Note

\title{
A Nonfermented Bread-Like Food Leavened by Microwave Heating
}

\author{
Kumi Chono, Yuka Itahara, Yoshimi Mochida, Naoko AKaI, Mihoko Tominaga and Kazuyoshi Sato* \\ Laboratory of Food Science, Faculty of Education, Hiroshima University 1-1-2 Kagamiyama, Higashi Hiroshima, 739-8523, Japan
}

Received August 28, 1997; Accepted June 9, 1998

\begin{abstract}
A nonfermented bread-like food, quick bread, was prepared using microwave heating in the leavening process and yogurt as the acidifying agent. The use of microwave heating effectively shortened the time of breadmaking, reduced the amount of sodium bicarbonate necessary as the leavening agent and increased the specific volume of the loaf. Yogurt used as the acidifying agent improved the loaf properties such as crust color and odor. The crumb grain of the bread-like food was more homogeneous in the loaf prepared by simultaneously heating three doughs than by heating a single dough. The highest evaluations in a sensory test were obtained for the loaf prepared using microwave heating with the addition of sodium bicarbonate and yogurt. Firming during brief storage was slower in the loaf containing these additives.
\end{abstract}

Keywords: nonfermented bread-like food, quick bread, microwave heating, leavening process, quick breadmaking

Various kinds of breads are prepared in the world, and flatbreads such as chapati, matzo and tortilla are known as unleavened breads (Coyle, 1982). For leavening, it is necessary to use yeast fermentation or chemical leavening agents. Both of these methods have their disadvantages; while fermentation is time-consuming and requires temperature control, chemical leavening produces a flavor deterioration through the use of ingredients such as sodium bicarbonate. In order to ameliorate these defects, it was decided to use microwave heating for the leavening process and acidifying agents.

Microwave heating has been used in the baking process for cakes (Lorenz et al., 1973; Stinson, 1986; Baker et al., 1990; Li \& Walker, 1996) and breads (Lorenz et al., 1973; Tsen, 1980, Ovadia \& Walker, 1995). Microwave baking is faster than conventional baking, but the products are known to have less acceptable characteristics, in particular a pale crust color (Stinson, 1986). Microwave ovens equipped with browning elements (Street \& Surratt, 1961), a microwave/convection oven (Stinson, 1986) or a microwave/air impingement oven (Li \& Walker, 1996) were found to improve the color development of the crust color during baking. However, few detailed studies have been made on the application of microwave heating to the leavening process during breadmaking.

The objective of our study was to prepare a nonfermented, bread-like food using both microwave oven heating in the leavening process and conventional oven heating in the baking process. We also studied the simultaneous use of sodium bicarbonate and acidifying agents such as yogurt in the leavening process in order to improve the color, odor and crumb grain of the loaf.

\footnotetext{
*To whom correspondence should be addressed.
}

\section{Materials and Methods}

The bread-like food was prepared based on the formula shown in Table 1. The materials are commercially available; wheat flour (hard flour) was obtained from Nisshin flour milling Co., Tokyo and margarine ("Royal chef") from the UCC Ueshima Coffee Co., Kobe. The yogurt used was a commercially available one ("Nachure," Snow Brand Milk Products Co., Tokyo) or prepared using Lactobacillus helveticus B-1 (formerly classified as Lactobacillus bulgaricus) or Streptococcus thermophilus as previously described (Yukitomo et al., 1984). Doughs were prepared according to a straight dough procedure. The materials other than margarine were mixed for $1 \mathrm{~min}$ and then kneaded by hand in a 1-1 plastic beaker followed by slowly adding the margarine for 5 min at ambient temperature. The dough was then rounded. The beaten yolk of an egg was brushed on the surface of the dough. About $90 \mathrm{~g}$ of dough was obtained from $50 \mathrm{~g}$ of wheat flour. As a control, the dough with beaten yolk brushed on the surface was baked in a conventional oven (National Electronic Oven NB 6603, Osaka) at $230^{\circ} \mathrm{C}$ for $15 \mathrm{~min}$, which we shall call the "conventional heating method." When microwave oven heating was used for the leavening process, the heating was performed for $1.5 \mathrm{~min}$, in the case of one dough, by placing it on the center of the plate of the oven $(2450 \mathrm{MHz}, 500 \mathrm{~W}$, National Microwave Oven NE-M315, Osaka) and for $3.5 \mathrm{~min}$, in the case of 3 doughs, by placing them at almost equal distances side by side on the plate. Immediately after, beaten yolk was brushed on the surface of each dough and they were then baked for 5 min using the conventional oven preheated at $230^{\circ} \mathrm{C}$. We shall call this process the "microwave heating method."

Weights and volumes of the bread-like foods were measured at $10 \mathrm{~min}$ after removal from the oven. The volume was measured by the rapeseed displacement method and the specific volume of the loaf was obtained using 3 to 7 determinations. Bread-like food firmness was measured with 
Table 1. Formula for bread-like food.

\begin{tabular}{lc}
\hline Materials & Flour basis (weight \%) \\
\hline Wheat flour & 100.0 \\
Sugar & 1.0 \\
Salt & 1.3 \\
Egg & 24.0 \\
Margarine & 20.0 \\
Distilled water & 33.0 \\
\hline Sodium bicarbonate & 0.25 \\
& 0.50 \\
Yogurt + Distilled water & $a$ ) \\
& $18.8+16.5$ \\
& $28.2+8.2$ \\
\hline
\end{tabular}

${ }^{a)}$ Distilled water is replaced by yogurt, assuming water content of yogurt as ca. $88 \%$.

a creep meter (Yamaden RE-3305, Tokyo) at 5 min, 1, 6 and $24 \mathrm{~h}$ after baking. The maximum stress (firmness) was measured by compression at a speed of $1 \mathrm{~mm} / \mathrm{s}$ to $18 \mathrm{~mm}$ from the surface of the loaf center with a plunger of $3 \mathrm{~mm}$ diameter (Yamaden plunger No. 3). Another bread-like food was cut vertically into two halves. The cross sections were qualitatively observed and photographed.

Sensory evaluation was performed by panelists consisting of 17 male and 17 female university students. Three types of bread-like food were used; i.e., one control sample containing no leavening agent, and two test samples, one containing sodium bicarbonate $(0.5 \%$ of wheat flour basis, weight \%) and one containing both sodium bicarbonate $(0.5 \%)$ and yogurt (37.6\%). These samples were prepared using the microwave heating method $(3.5 \mathrm{~min}$ microwave heating followed by 5 min baking), after which they were wrapped with a polyethylene sheet and allowed to stand for $1 \mathrm{~h}$. They were crosssectioned at the center and each piece was randomly presented to the panelists. Water was provided for them to rinse between samples. The samples were evaluated for taste, color, odor, firmness, crumb appearance and overall preference using a hedonic score card with 7 hedonic scale-points ranging from 1 (dislike very much) to 7 (like very much). The data were analyzed by a one-way analysis of variance (ANOVA). Means comparisons were made by Fisher's Least Significance Differences (LSD) test at the probability level of $p=0.05$.

\section{Results and Discussion}

The specific volume of the loaf was increased by adding sodium bicarbonate and microwave oven heating in the leavening process, i.e., the microwave heating method (Fig. 1). The increment of the specific volume was larger in the loaf using microwave heating than in the one to which sodium bicarbonate was added without microwave heating. This effect of microwave heating seems to be caused by expanding air and vaporizing moisture in the interior of the loaf, which tends to produce somewhat larger bubbles inside the loaf. In contrast, the addition of sodium bicarbonate reduced the large bubble formation, but deteriorated the flavor and the color when even a small amount, $0.25 \%$ sodium bicarbonate, was added.

To prevent the alkaline odor and the deterioration of crumb color, we examined the effects of acidic materials such

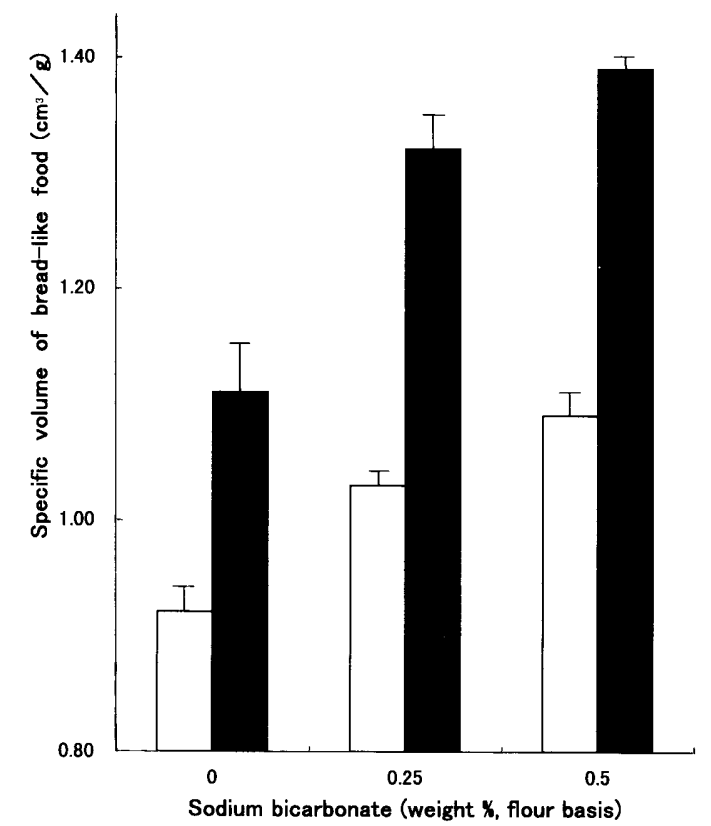

Fig. 1. Effect of sodium bicarbonate addition and microwave heating on specific volume of bread-like foods. $\square$ : conventional heating method, microwave heating method; means \pm S.D. of three replications.

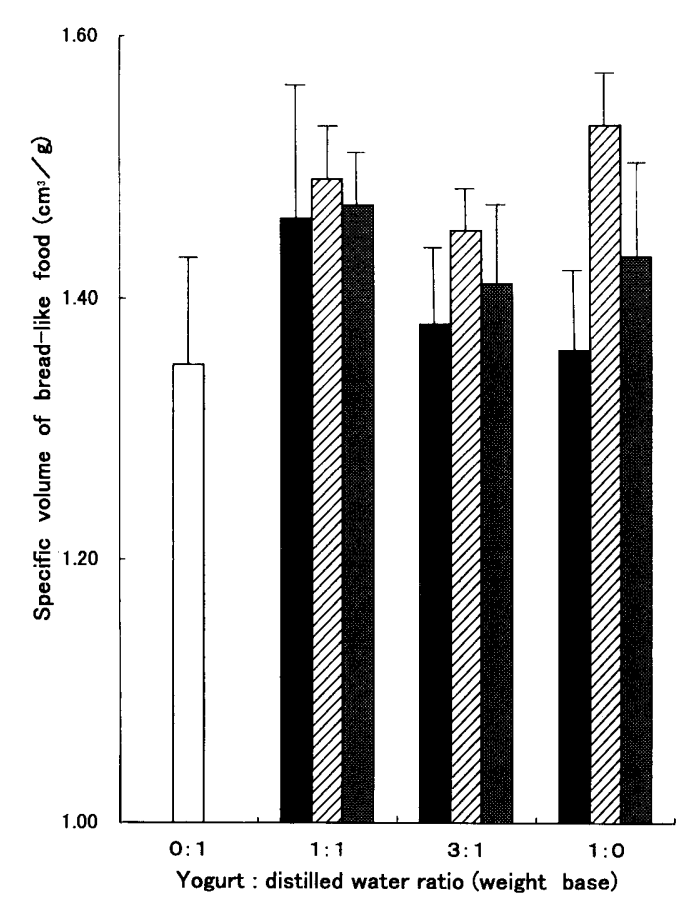

Fig. 2. Effect of additions of sodium bicarbonate ( $0.5 \%$ each) and yogurt on specific volume of bread-like foods in the microwave heating method. $\Pi$ : none, 口: L. helveticus-yogurt, $\mathbb{Z}$ : S. thermophilus-yogurt; means \pm S.D. of seven replications.

as fruit juices including lemon juice and yogurt. The effect of yogurt addition was superior to that of fruit juice addition (data not shown). The specific volume of the loaf changed depending on the amount of yogurt added and the species of the inoculated lactic acid bacteria (Fig. 2). In particular, yogurt obtained by inoculation of $S$. thermophilus $(S$. thermophilus-yogurt) had the greatest influence on the specific 
volume of the loaf, which was increased by the complete substitution of yogurt for water in the bread-like food preparation. The effective component of $S$. thermophilusyogurt is under investigation. In contrast, the specific volume was somewhat decreased by completely replacing water with L. helveticus-yogurt. This decrease in the specific volume appears to be due to the high acidity (lactic acid concentration in yogurt: ca. $1.5 \%$ ) brought about by $L$. helveticus, while the acidities of $S$. thermophilus-yogurt and the commercial yogurt were less than $1.0 \%$. The complete substitution of the commercial yogurt, which is assumed to contain both $L$. helveticus and $S$. thermophilus, only slightly changed the specific volume of the loaf. At any rate, the complete substitution of yogurt for water made it easy to prepare the doughs, and increased the volume. Furthermore, the substitution had favorable effects on the taste, odor and color of the loaf.

Heating three doughs at the same time with a microwave oven took a longer time (ca. $3.5 \mathrm{~min}$ ) than heating a single dough. Breads obtained from the heating of three doughs had better crumb grain appearance, but a slightly smaller volume. These effects were probably caused by the decrease in the microwave dose per dough. This seems to suggest that there is a suitable dose of microwave irradiation for making breadlike foods, which is a possible objective for further study.

Foods from wheat flour are known to firm after microwave heating (Higo \& Noguchi, 1987). We thus examined the time course of firming after baking of the bread-like food (Table 2). For the control from the conventional heating method, the crust firmed with baking and gave a fairy high value of maximum stress (firmness: $6.6 \times 10^{5} \mathrm{~N} / \mathrm{m}^{2}$ ). In contrast, the other control sample obtained by the microwave heating method for leavening did not firm very much just after baking, but the firming was very drastic (maximum stress after $24 \mathrm{~h}: 11.3 \times 10^{5} \mathrm{~N} / \mathrm{m}^{2}$ ). This marked firming was appreciably reduced by the addition of sodium bicarbonate or yogurt with sodium bicarbonate. The addition of monoglyceride

Table 2. Time course of firmness of bread-like foods after baking.

\begin{tabular}{|c|c|c|c|c|}
\hline \multirow{3}{*}{ Samples } & \multicolumn{4}{|c|}{ Time after baking } \\
\hline & $5 \mathrm{~min}$ & $1 \mathrm{~h}$ & $6 \mathrm{~h}$ & $24 \mathrm{~h}$ \\
\hline & \multicolumn{4}{|c|}{ Maximum stress $\left(\times 10^{-5} \mathrm{~N} / \mathrm{m}^{2}\right)$} \\
\hline Control $^{a}$ & 6.6 & 6.7 & 9.4 & 10.6 \\
\hline Control $^{b)}$ & 2.4 & 5.7 & 6.5 & 11.3 \\
\hline Sodium bicarbonate-supplemented ${ }^{b)}$ & 1.8 & 2.5 & 3.8 & 6.4 \\
\hline $\begin{array}{l}\text { Sodium bicarbonate and yogurt- } \\
\text { supplemented }{ }^{b)}\end{array}$ & 1.5 & 2.5 & 2.3 & 4.8 \\
\hline
\end{tabular}

${ }^{a)}$ Heating was done with the conventional heating method.

${ }^{b)}$ Heating was done with the microwave heating method. was reported to be effective for retardation of the retrogradation of starch and for firming of the bread (Yamauchi et al. 1993). Components such as the proteins of the additives in this study might also prevent interactions between starch molecules, but the detailed mechanism of the effect of additives like yogurt and other efficient materials for the prevention of firming remains to be studied.

Bread-like foods obtained by the microwave heating method puffed better than that prepared by the conventional heating method. The crumb grain became more homogeneous in the sample prepared with the addition of sodium bicarbonate and yogurt as shown in Fig. 3. Furthermore, whitening of the crumb color and dark-browning of the crust color increased.

Since the bread-like food prepared using the conventional
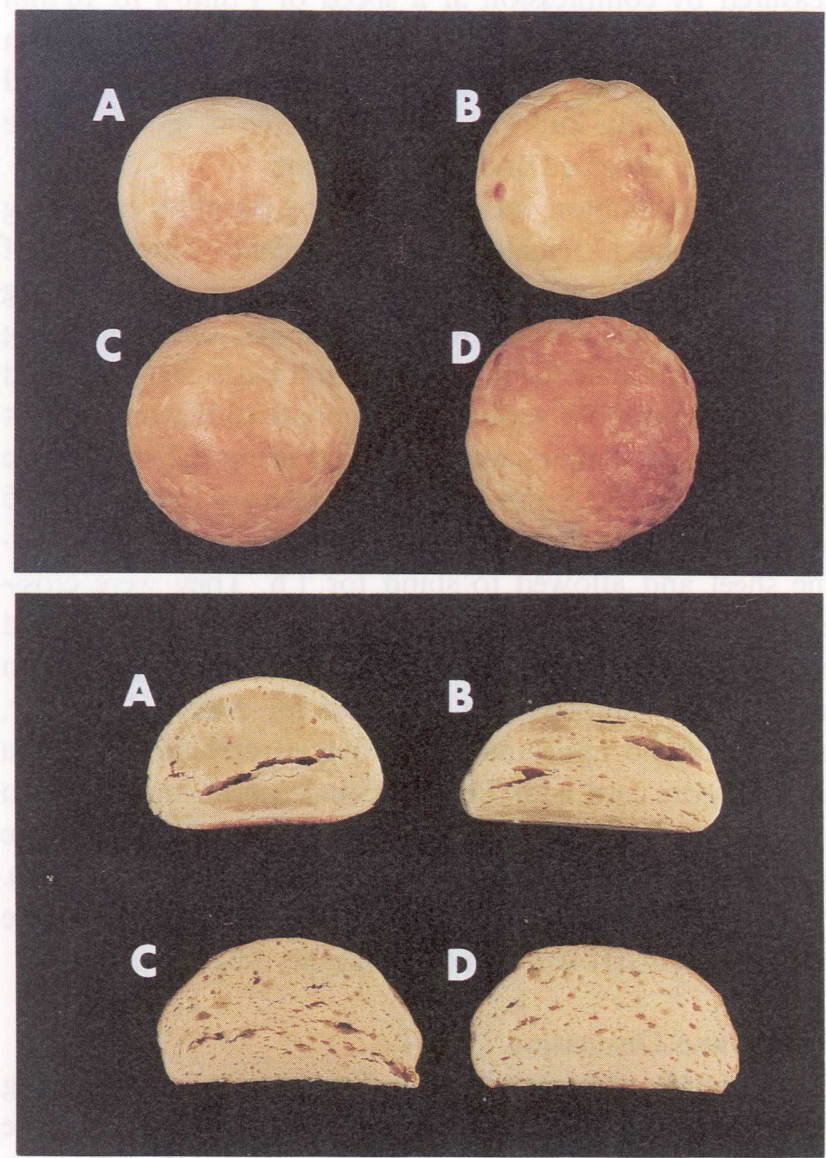

Fig. 3. External and cross-sectional appearances of bread-like foods. A: no additives, conventional heating method; B: no additives, microwave heating method; C: sodium bicarbonate, microwave heating method; D: sodium bicarbonate and yogurt, microwave heating method.

Table 3. Scores of bread-like foods in sensory evaluation.

\begin{tabular}{|c|c|c|c|c|c|c|}
\hline Additives & taste & color & odor & firmness & crumb grain & overall preference \\
\hline None (control) & $4.2^{a b} \pm 1.3$ & $4.5^{a} \pm 1.1$ & $4.2^{a} \pm 0.9$ & $4.2^{a} \pm 1.4$ & $3.9^{a} \pm 1.2$ & $4.1^{a b} \pm 1.3$ \\
\hline Sodium bicarbonate & $3.7^{b} \pm 1.6$ & $5.1^{b} \pm 1.0$ & $4.0^{a} \pm 1.5$ & $4.0^{a} \pm 1.4$ & $3.8^{a} \pm 1.2$ & $3.6^{a} \pm 1.2$ \\
\hline Sodium bicarbonate and yogurt & $4.6^{a} \pm 1.3$ & $5.6^{b} \pm 1.0$ & $5.0^{b} \pm 1.3$ & $4.5^{a} \pm 1.1$ & $4.5^{b} \pm 1.1$ & $4.6^{b} \pm 1.3$ \\
\hline
\end{tabular}

Values are mean \pm S.D. of 34 scores. The means in the same column with the same superscripts are not significantly different $(p<0.05)$. 
heating method exhibited different characteristics, e.g. firmness, from the other three samples prepared using the microwave heating method, sensory evaluation was conducted on the latter three samples (Table 3). The scores of the control were 3.9 to 4.5 in all sensory attributes, but the addition of sodium bicarbonate slightly lowered the scores except for color $(5.1 \pm 1.0)$, particularly the score for taste (3.7 \pm 1.6 ; dislike slightly) and overall preference (3.6 \pm 1.2$)$. There were negligible differences in gender with the exception of the score for color, which was significantly higher in females. The sample supplemented with sodium bicarbonate and yogurt had the highest scores (4.5 to 5.6; like slightly) and except for firmness, they were significantly different from the sample supplemented with only sodium bicarbonate. We assume that this bread-like food would be acceptable and this method would be useful for the preparation on a small scale in homes and schools, particularly as the method requires less additives such as baking powder which is necessary for ordinary quick bread (Dryer et al., 1982).

Acknowledgements This work was supported in part by a Grant-inAid for Scientific Research from the Ministry of Education, Science, Sports and Culture of Japan (No. 08878034).

\section{References}

Baker, B.A., Davis, E.A. and Gordon, J. (1990). Glass and metal pans for use with microwave- and conventionally heated cakes. Cereal
Chem., 67(5), 448-451.

Coyle, L.P. (1982). Bread. In "The World Encyclopedia of Food," Frances Printer (Publishers) Ltd., London, p. 98.

Dryer, S.B., Phillips, S.G., Powell, T.S., Uebersax, M.A. and Zabik, M. E. (1982). Dry roasted navy bean flour incorporation in a quick bread. Cereal Chem., 59(4), 319-320.

Higo, O. and Noguchi, S. (1987). Process of bread hardening by microwave-heating. J. Jpn. Soc. Food Sci. Technol, 34(12), 781787.

Li, A. and Walker, C.E. (1996). Cake baking in conventional, impingement and hybrid ovens. J. Food Sci., 61(1), 188-197.

Lorenz, K., Charman, E. and Dilsaver, W. (1973). Baking with microwave energy. Food Technol, 27(12), 28-30, 32, 34, 36.

Ovadia, D.Z. and Walker, C.E. (1995). Microwave baking of bread. $J$. Microwave Power Electromagn. Energy, 30(2), 81-89.

Stinson, C.T. (1986). Effects of microwave/convection baking and pan characteristics on cake quality. J. Food. Sci, 51(6), 1580-1582.

Street, M.B. and Surratt, H.K. (1961). The effect of electronic cookery upon the appearance and platability of yellow cake. J. Home Econ., 53(4), 285-291.

Tsen, C.C. (1980). Microwave energy for bread baking and its effect on the nutritive value of bread: a review. J. Food Protect., 41(8), 638640.

Yamauchi, H., Kanesige, H., Fujimura, M., Hashimoto, S., Oya, K., Hirakawa, K. and Kobayashi, T. (1993). Role of starch and gluten on staling of white bread treated with microwave-heating. J. Jpn. Soc. Food Sci. Technol., 40(1), 42-51.

Yukitomo, K., Sado, Y., Tanaka, Y., Nakanishi, F. and Sato, K. (1984). Change in vitamin B12 content during lactic acid fermentation of milk. J. Home Econ. Jpn., 35(9), 626-629. 